\title{
On the Spectrum and Spectral Norms of $r$-Circulant Matrices with Generalized $k$-Horadam Numbers Entries
}

\author{
Lele Liu \\ College of Science, University of Shanghai for Science and Technology, Shanghai 200093, China \\ Correspondence should be addressed to Lele Liu; ahhylau@163.com
}

Received 17 May 2014; Accepted 25 August 2014; Published 31 August 2014

Academic Editor: Chengpeng Bi

Copyright (C) 2014 Lele Liu. This is an open access article distributed under the Creative Commons Attribution License, which permits unrestricted use, distribution, and reproduction in any medium, provided the original work is properly cited.

This work is concerned with the spectrum and spectral norms of $r$-circulant matrices with generalized $k$-Horadam numbers entries. By using Abel transformation and some identities we obtain an explicit formula for the eigenvalues of them. In addition, a sufficient condition for an $r$-circulant matrix to be normal is presented. Based on the results we obtain the precise value for spectral norms of normal $r$-circulant matrix with generalized $k$-Horadam numbers, which generalize and improve the known results.

\section{Introduction}

There is no doubt that the $r$-circulant matrices have been one of the most interesting research areas in computation mathematics. It is well known that these matrices have a wide range of applications in signal processing, digital image disposal, coding theory, linear forecast, and design of selfregress.

There are many works concerning estimates for spectral norms of $r$-circulant matrices with special entries. For example, Solak [1] established lower and upper bounds for the spectral norms of circulant matrices with Fibonacci and Lucas numbers entries. subsequently, Ipek [2] investigated some improved estimations for spectral norms of these matrices. Bani-Domi and Kittaneh [3] established two general norm equalities for circulant and skew circulant operator matrices. Shen and Cen [4] gave the bounds of the spectral norms of $r$-circulant matrices whose entries are Fibonacci and Lucas numbers. In [5] they defined $r$-circulant matrices involving $k$-Lucas and $k$-Fibonacci numbers and also investigated the upper and lower bounds for the spectral norms of these matrices.

Recently, Yazlik and Taskara [6] define a generalization $\left\{H_{k, n}\right\}$ of the special second-order sequences such as Fibonacci, Lucas, $k$-Fibonacci, $k$-Lucas, generalized $k$ Fibonacci and $k$-Lucas, Horadam, Pell, Jacobsthal, and Jacobsthal-Lucas sequences. For any integer number $k \geqslant 1$, the generalized $k$-Horadam sequence $\left\{H_{k, n}\right\}$ is defined by the following recursive relation:

$$
\begin{gathered}
H_{k, n+2}=f(k) H_{k, n+1}+g(k) H_{k, n}, \\
H_{k, 0}=a, \quad H_{k, 1}=b,
\end{gathered}
$$

where $f(k)$ and $g(k)$ are scaler-value polynomials, $f^{2}(k)+$ $4 g(k)>0$. The following are some particular cases.

(i) If $f(k)=k, g(k)=1$ and $a=0, b=1$, the $k$-Fibonacci sequence is obtained:

$$
F_{k, n+2}=k F_{k, n+1}+F_{k, n}, \quad F_{k, 0}=0, \quad F_{k, 1}=1 .
$$

(ii) If $f(k)=k, g(k)=1$ and $a=2, b=k$, the $k$-Lucas sequence is obtained:

$$
L_{k, n+2}=k L_{k, n+1}+L_{k, n}, \quad F_{k, 0}=0, \quad F_{k, 1}=k .
$$

(iii) If $f(k)=1, g(k)=1$ and $a=0, b=1$, the Fibonacci sequence is obtained:

$$
F_{n+2}=F_{n+1}+F_{n}, \quad F_{0}=0, \quad F_{1}=1 .
$$

(iv) If $f(k)=1, g(k)=1$ and $a=2, b=1$, the Lucas sequence is obtained:

$$
L_{n+2}=L_{n+1}+L_{n}, \quad L_{0}=2, \quad L_{1}=1 .
$$


(v) If $f(k)=1, g(k)=2$ and $a=0, b=1$, the Jacobsthal sequence is obtained:

$$
J_{n+2}=J_{n+1}+2 J_{n}, \quad J_{0}=0, \quad J_{1}=1 .
$$

In [7], the authors present new upper and lower bounds for the spectral norm of an $r$-circulant matrix $C_{r}\left(H_{k, 0}, H_{k, 1}, \ldots, H_{k, n-1}\right)$, and they study the spectral norm of circulant matrix with generalized $k$-Horadam numbers in [8]. In this paper, we first give an explicit formula for the eigenvalues of $r$-circulant matrix with generalized $k$-Horadam numbers entries using different methods in [7]. Afterwards, we present a sufficient condition for an $r$-circulant matrix to be normal. Based on the results, the precise value for spectral norms of normal $r$-circulant matrix whose entries are generalized $k$-Horadam numbers is obtained, which generalize and improve the main results in $[1,2,4,5]$.

\section{Preliminaries}

In this section, we present some known lemmas and results that will be used in the following study.

Definition 1. For any given $c_{0}, c_{1}, \ldots, c_{n-1} \in \mathbb{C}$, the $r$-circulant matrix $C$, denoted by $C=C_{r}\left(c_{0}, c_{1}, \ldots, c_{n-1}\right)$, is of the form

$$
\left(\begin{array}{ccccc}
c_{0} & c_{1} & c_{2} & \cdots & c_{n-1} \\
r c_{n-1} & c_{0} & c_{1} & \cdots & c_{n-2} \\
r c_{n-2} & r c_{n-1} & c_{0} & \cdots & c_{n-3} \\
\vdots & \vdots & \vdots & \ddots & \vdots \\
r c_{1} & r c_{2} & r c_{3} & \cdots & c_{0}
\end{array}\right)
$$

It is obvious that the matrix $C_{r}$ turns into a classical circulant matrix for $r=1$.

Lemma 2 (see [9]). Let $C=C_{r}\left(c_{0}, c_{1}, \ldots, c_{n-1}\right)$ be an $r$ circulant matrix; then the eigenvalues of $C$ are given by

$$
\lambda_{i}=\sum_{j=0}^{n-1} c_{j} \mu_{i}^{j}, \quad \mu_{i}=r^{1 / n} \omega^{i}, \quad i=0,1, \ldots, n-1,
$$

where $\omega=e^{-2 \pi i / n}$ is the $n$th root of unity.

Let us take any matrix $A=\left[a_{i j}\right]$ of order $n$; it is well known that the spectral norm of matrix $A$ is

$$
\|A\|_{2}=\sqrt{\max _{0 \leqslant i \leqslant n-1} \lambda_{i}\left(A^{H} A\right)}
$$

where $A^{H}$ is the conjugate transpose of $A$ and $\lambda_{i}\left(A^{H} A\right)$ is the eigenvalue of $A^{H} A$.

For a normal matrix $A$ (i.e., $A A^{H}=A^{H} A$ ), we have the following lemma.

Lemma 3 (see [10]). Let $A$ be a normal matrix with eigenvalues $\lambda_{0}, \lambda_{1}, \ldots, \lambda_{n-1}$. Then the spectral norm of $A$ is

$$
\|A\|_{2}=\max _{0 \leqslant i \leqslant n-1}\left|\lambda_{i}\right|
$$

The following lemma can be found in [11].

Lemma 4 (see [11], Abel transformation). Suppose that $\left\{a_{i}\right\}$ and $\left\{b_{i}\right\}$ are two sequences, and $S_{i}=a_{1}+a_{2}+\cdots+a_{i}(i=$ $1,2, \ldots)$; then

$$
\sum_{i=1}^{n} a_{i} b_{i}=S_{n} b_{n}-\sum_{i=1}^{n-1}\left(b_{i+1}-b_{i}\right) S_{i}
$$

\section{Spectrum of $r$-Circulant Matrix with Generalized $k$-Horadam Numbers}

We start this section by giving the following lemma.

Lemma 5. Suppose that $\left\{H_{k, i}\right\}_{i \in \mathbb{N}}$ is a generalized $k$-Horadam sequence defined in (1). The following conclusions hold.

(1) If $f(k)+g(k) \neq 1$, then

$$
\sum_{i=0}^{n} H_{k, i}=\frac{H_{k, n+1}+g(k) H_{k, n}+f(k) a-a-b}{f(k)+g(k)-1} .
$$

(2) If $f(k)+g(k)=1$, then

$$
\sum_{i=0}^{n} H_{k, i}=\frac{g(k) H_{k, n}+n[g(k) a+b]+a}{g(k)+1} .
$$

Proof. (1) According to (1), we have

$$
\sum_{i=0}^{n} H_{k, i}=f(k) \sum_{i=0}^{n} H_{k, i-1}+g(k) \sum_{i=0}^{n} H_{k, i-2} .
$$

Changing the summation index in (14), we have

$$
\begin{aligned}
\sum_{i=0}^{n} H_{k, i} & \\
= & f(k)\left(\sum_{i=0}^{n} H_{k, i}-H_{k, n}+H_{k,-1}\right) \\
& +g(k)\left(\sum_{i=0}^{n} H_{k, i}-H_{k, n-1}-H_{k, n}+H_{k,-1}+H_{k,-2}\right) .
\end{aligned}
$$

By direct calculation, together with recursive relation (1), one can obtain that

$$
\begin{aligned}
& {[f(k)+g(k)-1] \sum_{i=0}^{n} H_{k, i}} \\
& =H_{k, n+1}+g(k) H_{k, n}+f(k) a-a-b .
\end{aligned}
$$

Therefore we immediately obtain (12) from $f(k)+g(k) \neq 1$.

(2) Suppose that $f(k)+g(k)=1$; we first illustrate that $H_{k, i+1}+g(k) H_{k, i} \equiv g(k) a+b$. Let $V_{i}=H_{k, i+1}+g(k) H_{k, i}$; then $V_{0}=g(k) a+b$. Combining (1) and $f(k)+g(k)=$ 1 , one can obtain that

$$
\begin{aligned}
V_{i+1} & =H_{k, i+2}+g(k) H_{k, i+1} \\
& =\left(f(k) H_{k, i+1}+g(k) H_{k, i}\right)+g(k) H_{k, i+1} \\
& =H_{k, i+1}+g(k) H_{k, i}=V_{i},
\end{aligned}
$$


which shows that $\left\{V_{i}\right\}$ is a constant sequence, and therefore

$$
H_{k, i+1}+g(k) H_{k, i}=V_{i}=V_{0}=g(k) a+b .
$$

Evaluating summation from 0 to $n$, we have

$$
\sum_{i=0}^{n} H_{k, i+1}+g(k) \sum_{i=0}^{n} H_{k, i}=(n+1)[g(k) a+b] .
$$

Changing the summation index in (19) gives

$$
\begin{gathered}
\left(\sum_{i=0}^{n} H_{k, i}+H_{k, n+1}-a\right)+g(k) \sum_{i=0}^{n} H_{k, i} \\
=(n+1)[g(k) a+b] .
\end{gathered}
$$

Therefore

$$
[g(k)+1] \sum_{i=0}^{n} H_{k, i}=g(k) H_{k, n}+n[g(k) a+b]+a .
$$

In view of assumptions $f^{2}(k)+g(k)>1$ and $f(k)+g(k)=1$, we know that $g(k)+1 \neq 0$. Thus we obtain (13) from (21).

From Lemma 5 we have the following theorem.

Theorem 6. Let $A=C_{r}\left(H_{k, 0}, H_{k, 1}, \ldots, H_{k, n-1}\right)$ be an $r$ circulant matrix with eigenvalues $\lambda_{0}, \lambda_{1}, \ldots, \lambda_{n-1}$; then for $i=$ $0,1,2, \ldots, n-1$ the following hold.

(1) If $f(k)+g(k) \neq 1$, then

$$
\begin{aligned}
\lambda_{i}=( & r H_{k, n}+g(k) r^{1+(1 / n)} \omega^{i} H_{k, n-1} \\
& \left.+r^{1 / n}[f(k) a-b] \omega^{i}-a\right) \\
& \times\left(r^{1 / n} \omega^{i} f(k)+r^{2 / n} \omega^{2 i} g(k)-1\right)^{-1} .
\end{aligned}
$$

(2) If $f(k)+g(k)=1$, then

$$
\begin{aligned}
\lambda_{i}= & \left(\left(g(k) r H_{k, n-1}+a\right)\left(1-r^{1 / n} \omega^{i}\right)\right. \\
& \left.+[g(k) a+b]\left(r^{1 / n} w^{i}-r\right)\right) \\
& \times\left(\left(1-r^{1 / n} \omega^{i}\right)\left[g(k) r^{1 / n} \omega^{i}+1\right]\right)^{-1} .
\end{aligned}
$$

Proof. According to Lemma 2, we have

$$
\lambda_{i}=\sum_{i=0}^{n-1} H_{k, i} \mu_{i}^{j}, \quad \mu_{i}=r^{1 / n} w^{i}
$$

Using Abel transformation (Lemma 4), we have

$$
\begin{aligned}
\lambda_{i} & =\mu_{i}^{n-1} \sum_{j=0}^{n-1} H_{k, j}-\sum_{j=0}^{n-2}\left(\left(\mu_{i}^{j+1}-\mu_{i}^{j}\right) \sum_{s=0}^{j} H_{k, s}\right) \\
& =\mu_{i}^{n-1} \sum_{j=0}^{n-1} H_{k, j}-\left(\mu_{i}-1\right) \sum_{j=0}^{n-2}\left(\mu_{i}^{j} \sum_{s=0}^{j} H_{k, s}\right) .
\end{aligned}
$$

(1) In the light of (12) and (25), one can obtain that

$$
\begin{aligned}
\lambda_{i}= & \mu_{i}^{n-1} \sum_{j=0}^{n-1} H_{k, j}-\frac{\mu_{i}-1}{f(k)+g(k)-1} \\
& \times \sum_{j=0}^{n-2} \mu_{i}^{j}\left[H_{k, j+1}+g(k) H_{k, j}+f(k) a-a-b\right] \\
= & \mu_{i}^{n-1} \frac{H_{k, n}+g(k) H_{k, n-1}+f(k) a-a-b}{f(k)+g(k)-1} \\
& -\frac{\mu_{i}-1}{f(k)+g(k)-1}
\end{aligned}
$$

$$
\begin{gathered}
\times\left(\sum_{j=0}^{n-2} H_{k, j+1} \mu_{i}^{j}+g(k) \sum_{j=0}^{n-2} H_{k, j} \mu_{i}^{j}\right. \\
\left.+[f(k) a-a-b] \sum_{j=0}^{n-2} \mu_{i}^{j}\right) .
\end{gathered}
$$

It is clear that

$$
\begin{aligned}
\sum_{j=0}^{n-2} H_{k, j+1} \mu_{i}^{j} & =\frac{\lambda_{i}-a}{\mu_{i}}, \\
\sum_{j=0}^{n-2} H_{k, j} \mu_{i}^{j} & =\lambda_{i}-\mu_{i}^{n-1} H_{k, n-1} .
\end{aligned}
$$


Substituting (27) into (26), we obtain that

$$
\begin{aligned}
& \lambda_{i}=\mu_{i}^{n-1} \frac{H_{k, n}+g(k) H_{k, n-1}+f(k) a-a-b}{f(k)+g(k)-1} \\
& -\frac{\mu_{i}-1}{f(k)+g(k)-1} \\
& \times\left(\frac{\lambda_{i}-a}{\mu_{i}}+g(k)\left(\lambda_{i}-\mu_{i}^{n-1} H_{k, n-1}\right)\right. \\
& \left.+[f(k) a-a-b] \sum_{j=0}^{n-2} \mu_{i}^{j}\right) \\
& =\frac{\left(1-\mu_{i}\right)\left[1+g(k) \mu_{i}\right]}{\mu_{i}[f(k)+g(k)-1]} \lambda_{i} \\
& +\frac{\mu_{i}^{n-1} H_{k, n}+\mu_{i}^{n} g(k) H_{k, n-1}}{f(k)+g(k)-1} \\
& +\frac{\mu_{i}^{n}(f(k) a-a-b)-a\left(1-\mu_{i}\right)}{\mu_{i}[f(k)+g(k)-1]} \\
& +\frac{\left(1-\mu_{i}^{n-1}\right)(f(k) a-a-b)}{f(k)+g(k)-1} \text {. }
\end{aligned}
$$

Therefore we have

$$
\begin{aligned}
& {\left[g(k) \mu_{i}^{2}+f(k) \mu_{i}-1\right] \lambda_{i} } \\
&=\mu_{i}^{n} H_{k, n}+g(k) \mu_{i}^{n+1} H_{k, n-1}+\mu_{i}^{n}(f(k) a-a-b) \\
&-a\left(1-\mu_{i}\right)+\left(\mu_{i}-r\right)(f(k) a-a-b) \\
&= r H_{k, n}+g(k) r^{1+(1 / n)} \omega^{i} H_{k, n-1} \\
&+r^{1 / n}[f(k) a-b] \omega^{i}-a .
\end{aligned}
$$

We immediately obtain formula (22) from (29).

(2) Taking into account (13) and (25), we have

$$
\begin{aligned}
\lambda_{i}= & \mu_{i}^{n-1} \sum_{j=0}^{n-1} H_{k, j}-\frac{\mu_{i}-1}{g(k)+1} \\
& \times \sum_{j=0}^{n-2} \mu_{i}^{j}\left[g(k) H_{k, j}+j \cdot(g(k) a+b)+a\right] \\
= & \mu_{i}^{n-1} \sum_{j=0}^{n-1} H_{k, j}+\frac{g(k)\left(1-\mu_{i}\right)}{g(k)+1} \\
& \times \sum_{j=0}^{n-2} H_{k, j} \mu_{i}^{j}+\frac{1-\mu_{i}}{g(k)+1} \sum_{j=0}^{n-2}[j \cdot(g(k) a+b)+a] \mu_{i}^{j}
\end{aligned}
$$

$$
\begin{aligned}
& =\frac{\mu_{i}^{n-1}\left[g(k) H_{k, n-1}+(n-1)(g(k) a+b)+a\right]}{g(k)+1} \\
& +\frac{g(k)\left(1-\mu_{i}\right)}{g(k)+1}\left(\lambda_{i}-H_{k, n-1} \mu_{i}^{n-1}\right) \\
& +\frac{1-\mu_{i}}{g(k)+1}[((n-2)(g(k) a+b)+a) \\
& \left.\quad \times \sum_{j=0}^{n-2} \mu_{i}^{j}-(g(k) a+b) \sum_{j=0}^{n-3} \sum_{s=0}^{j} \mu_{i}^{s}\right] .
\end{aligned}
$$

It follows that

$$
\begin{aligned}
\lambda_{i}= & \frac{g(k) \mu_{i}^{n}}{g(k)+1} H_{k, n-1}+\frac{g(k)\left(1-\mu_{i}\right)}{g(k)+1} \lambda_{i} \\
& +\frac{\mu_{i}^{n-1}[(n-1)(g(k) a+b)+a]}{g(k)+1} \\
& +\frac{1-\mu_{i}}{g(k)+1}[((n-2)(g(k) a+b)+a) \\
= & \left.\frac{g(k)\left(1-\mu_{i}\right)}{g(k)+1} \lambda_{i} \sum_{j=0}^{n-2} \mu_{i}^{j}-\frac{(g(k) a+b)}{1-\mu_{i}} \sum_{j=0}^{n-3}\left(1-\mu_{i}^{j+1}\right)\right] \\
& +\frac{g(k) \mu_{i}^{n} H_{k, n-1}+\mu_{i}^{n-1}(g(k) a+b)+a}{g(k)+1} \\
& +\frac{(g(k) a+b)\left(\mu_{i}-\mu_{i}^{n}\right)}{(g(k)+1)\left(1-\mu_{i}\right)} .
\end{aligned}
$$

Therefore we obtain (23). This concludes the proof.

\section{Spectral Norms of Normal $r$-Circulant Matrices}

In this section, we consider the spectral norms of normal $r$ circulant matrix whose entries are generalized $k$-Horadam numbers. Our results generalize and improve the results in $[1,2,4,5]$. The following lemma can be found in [9], and we give a concise proof.

Lemma 7. Let $A=C_{r}\left(a_{0}, a_{1}, \ldots, a_{n-1}\right)$ be an $r$-circulant matrix. If $|r|=1$, then $A$ is normal matrix.

Proof. It is well known that

$$
A=\sum_{i=0}^{n-1} a_{i} P^{i}, \quad P=\left(\begin{array}{cc}
0 & I_{n-1} \\
r & 0
\end{array}\right) .
$$


If $|r|=1$, then

$$
P P^{H}=\left(\begin{array}{cc}
0 & I_{n-1} \\
r & 0
\end{array}\right)\left(\begin{array}{cc}
0 & \bar{r} \\
I_{n-1} & 0
\end{array}\right)=I_{n}
$$

That is, $P^{H}=P^{-1}$. According to (32), we obtain that

$$
\begin{aligned}
A A^{H} & =\left(\sum_{i=0}^{n-1} a_{i} P^{i}\right)\left(\sum_{j=0}^{n-1} a_{j}\left(P^{H}\right)^{j}\right) \\
& =\left(\sum_{i=0}^{n-1} a_{i} P^{i}\right)\left(\sum_{j=0}^{n-1} a_{j}\left(P^{-1}\right)^{j}\right)=\sum_{i=0}^{n-1} \sum_{j=0}^{n-1} a_{i} a_{j} P^{i-j},
\end{aligned}
$$

$$
\begin{aligned}
A^{H} A & =\left(\sum_{j=0}^{n-1} a_{j}\left(P^{H}\right)^{j}\right)\left(\sum_{i=0}^{n-1} a_{i} P^{i}\right) \\
& =\left(\sum_{j=0}^{n-1} a_{j} P^{-j}\right)\left(\sum_{i=0}^{n-1} a_{j} P^{i}\right)=\sum_{i=0}^{n-1} \sum_{j=0}^{n-1} a_{i} a_{j} P^{i-j} .
\end{aligned}
$$

Therefore $A A^{H}=A^{H} A$, which shows that $A$ is normal.

According to Theorem 6 and Lemma 7, we have the following theorem.

Theorem 8. Suppose that $A=C_{r}\left(H_{k, 0}, H_{k, 1}, \ldots, H_{k, n-1}\right)$ is an $r$-circulant matrix. If $|r|=1$ and $H_{k, i} \geqslant 0, i=0,1,2, \ldots, n-1$, then the spectral norm of $A$ is

$$
\|A\|_{2}=\left\{\begin{array}{lll}
\max _{0 \leqslant i \leqslant n-1}\left|\frac{r H_{k, n}+g(k) r^{1+(1 / n)} \omega^{i} H_{k, n-1}+r^{1 / n}[f(k) a-b] \omega^{i}-a}{r^{1 / n} \omega^{i} f(k)+r^{2 / n} \omega^{2 i} g(k)-1}\right|, & f(k)+g(k) \neq 1, \\
\max _{0 \leqslant i \leqslant n-1}\left|\frac{\left(g(k) r H_{k, n-1}+a\right)\left(1-r^{1 / n} \omega^{i}\right)+[g(k) a+b]\left(r^{1 / n} w^{i}-r\right)}{\left(1-r^{1 / n} \omega^{i}\right)\left[g(k) r^{1 / n} \omega^{i}+1\right]}\right|, & f(k)+g(k)=1 .
\end{array}\right.
$$

The following theorem simplifies and generalizes the results of Theorem 2.2 in [12].

Theorem 9. Let $A=\operatorname{Circ}\left(H_{k, 0}, H_{k, 1}, \ldots, H_{k, n-1}\right)$ be a circulant matrix; then

$$
\|A\|_{2}=\left\{\begin{array}{r}
\frac{H_{k, n}+g(k) H_{k, n-1}+f(k) a-a-b}{f(k)+g(k)-1}, \\
f(k)+g(k) \neq 1, \\
\frac{g(k) H_{k, n-1}+(n-1)[g(k) a+b]+a}{g(k)+1}, \\
f(k)+g(k)=1 .
\end{array}\right.
$$

Proof. Suppose that $r=1$; it follows from Lemma 7 that $A$ is normal. Notice that

$$
\left|\lambda_{i}\right|=\left|\sum_{i=0}^{n-1} H_{k, i} \mu_{i}^{j}\right| \leqslant \sum_{i=0}^{n-1} H_{k, i}\left|\mu_{i}\right|^{j}=\lambda_{0} .
$$

It follows from Lemma 3 that $\|A\|_{2}=\lambda_{0}$. According to Theorem 6 , if $f(k)+g(k) \neq 1$ and $r=1$, we obtain that

$$
\|A\|_{2}=\lambda_{0}=\frac{H_{k, n}+g(k) H_{k, n-1}+f(k) a-a-b}{f(k)+g(k)-1} .
$$

Similarly, if $f(k)+g(k)=1$, it follows that

$$
\|A\|_{2}=\lambda_{0}=\frac{g(k) H_{k, n-1}+(n-1)[g(k) a+b]+a}{g(k)+1} .
$$

This completes the proof.
Taking into account formulae (4)-(6), we have the following corollary.

Corollary 10. Let $A_{1}=\operatorname{Circ}\left(F_{0}, F_{1}, \ldots, F_{n-1}\right)$ be a circulant matrix; then

$$
\left\|A_{1}\right\|_{2}=F_{n+1}-1 .
$$

Corollary 11. Let $A_{2}=\operatorname{Circ}\left(L_{0}, L_{1}, \ldots, L_{n-1}\right)$ be a circulant matrix; then

$$
\left\|A_{2}\right\|_{2}=F_{n+2}+F_{n}-1 .
$$

Corollary 12. Let $A_{3}=\operatorname{Circ}\left(J_{0}, J_{1}, \ldots, J_{n-1}\right)$ be a circulant matrix; then

$$
\left\|A_{3}\right\|_{2}=\frac{J_{n+1}-1}{2}
$$

\section{Conflict of Interests}

The author declares that there is no conflict of interests regarding the publication of this paper.

\section{References}

[1] S. Solak, "On the norms of circulant matrices with the Fibonacci and Lucas numbers," Applied Mathematics and Computation, vol. 160, no. 1, pp. 125-132, 2005.

[2] A. Ipek, "On the spectral norms of circulant matrices with classical Fibonacci and Lucas numbers entries," Applied Mathematics and Computation, vol. 217, no. 12, pp. 6011-6012, 2011. 
[3] W. Bani-Domi and F. Kittaneh, "Norm equalities and inequalities for operator matrices," Linear Algebra and Its Applications, vol. 429, no. 1, pp. 57-67, 2008.

[4] S. Shen and J. Cen, "On the bounds for the norms of $r$ circulant matrices with the Fibonacci and Lucas numbers," Applied Mathematics and Computation, vol. 216, no. 10, pp. 2891-2897, 2010.

[5] S. Shen and J. Cen, "On the spectral norms of r-circulant matrices with the k-Fibonacci and k-Lucas numbers," International Journal of Contemporary Mathematical Sciences, vol. 5, no. 912, pp. 569-578, 2010.

[6] Y. Yazlik and N. Taskara, "A note on generalized $k$-Horadam sequence," Computers \& Mathematics with Applications, vol. 63, no. 1, pp. 36-41, 2012.

[7] Y. Yazlik and N. Taskara, "On the norms of an $r$-circulant matrix with the generalized $k$-Horadam numbers," Journal of Inequalities and Applications, vol. 2013, article 394, 2013.

[8] Y. Yazlik and N. Taskara, "Spectral norm, eigenvalues and determinant of circulant matrix involving the generalized $k$ Horadam numbers," Ars Combinatoria, vol. 104, pp. 505-512, 2012.

[9] Z. Jiang and Z. Zhou, "Nonsingularity of $r$-circulant matrices," Applied Mathematics: A Journal of Chinese Universities, vol. 10, no. 2, pp. 222-226, 1995.

[10] R. A. Horn and C. R. Johnson, Matrix Analysis, Cambridge University Press, 1985.

[11] W. Rudin, Principles of Mathematical Analysis, McGraw-Hill, 3rd edition, 1976.

[12] E. G. Kocer, T. Mansour, and N. Tuglu, "Norms of circulant and semicirculant matrices with Horadam's numbers," Ars Combinatoria, vol. 85, pp. 353-359, 2007. 


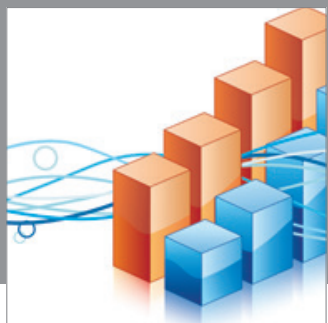

Advances in

Operations Research

mansans

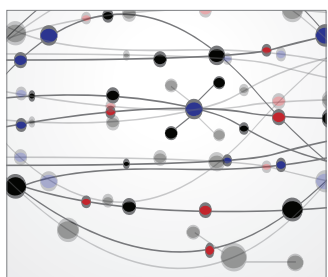

The Scientific World Journal
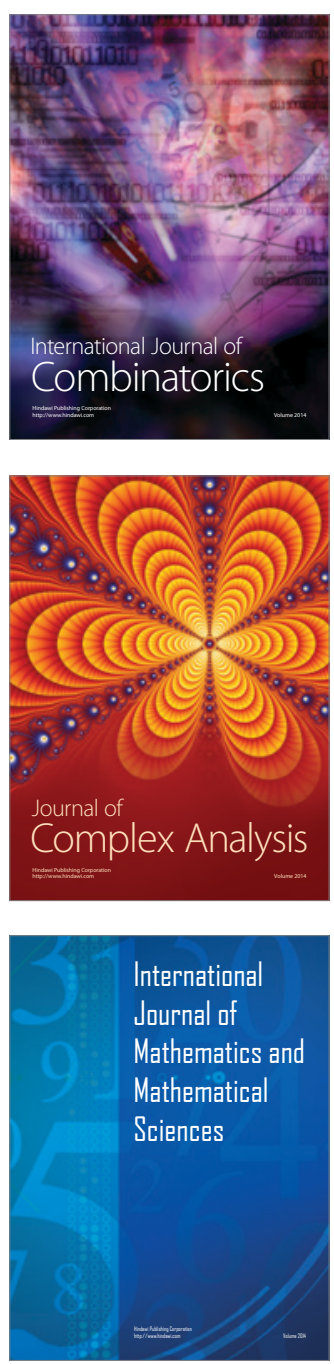
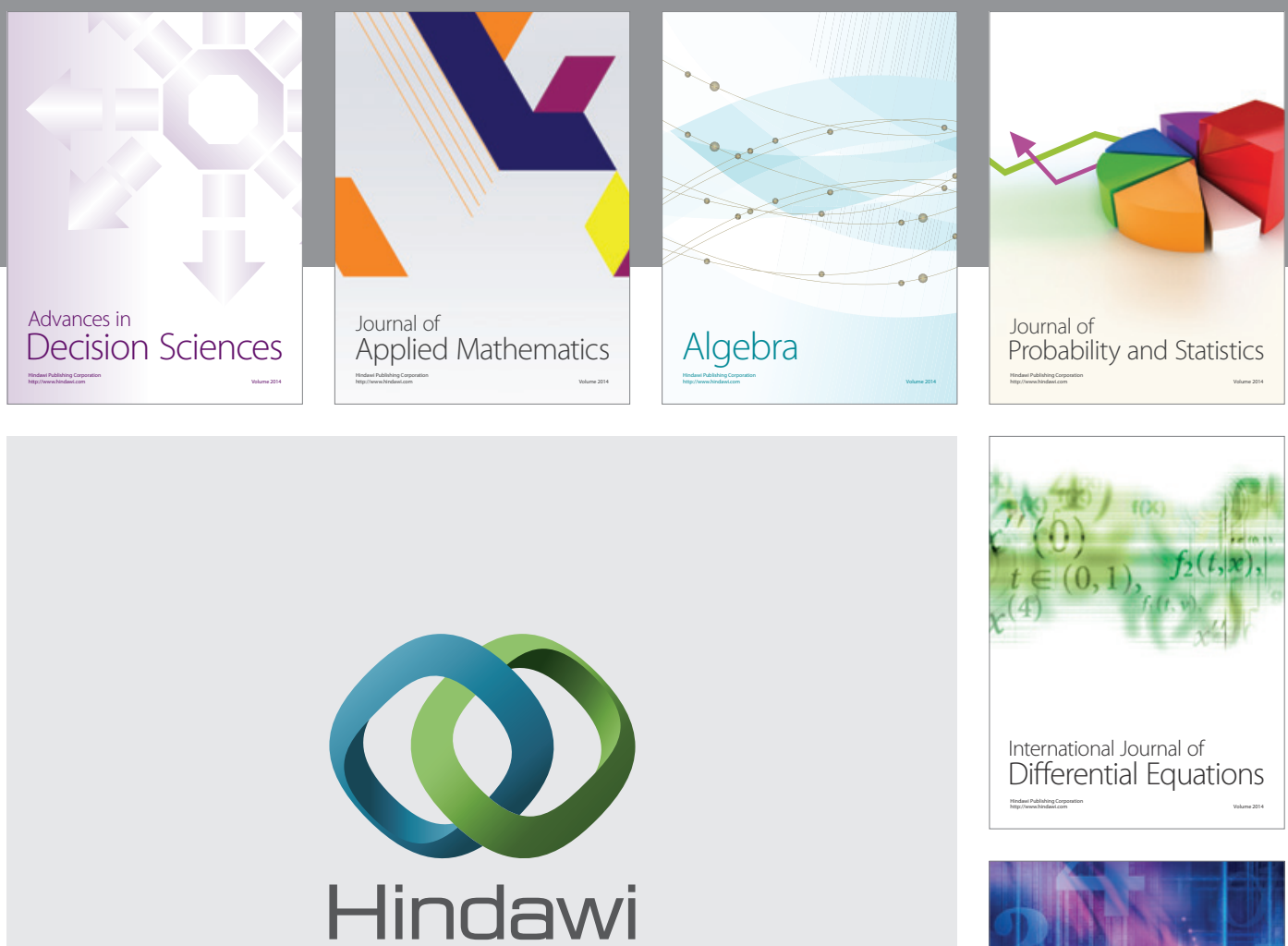

Submit your manuscripts at http://www.hindawi.com
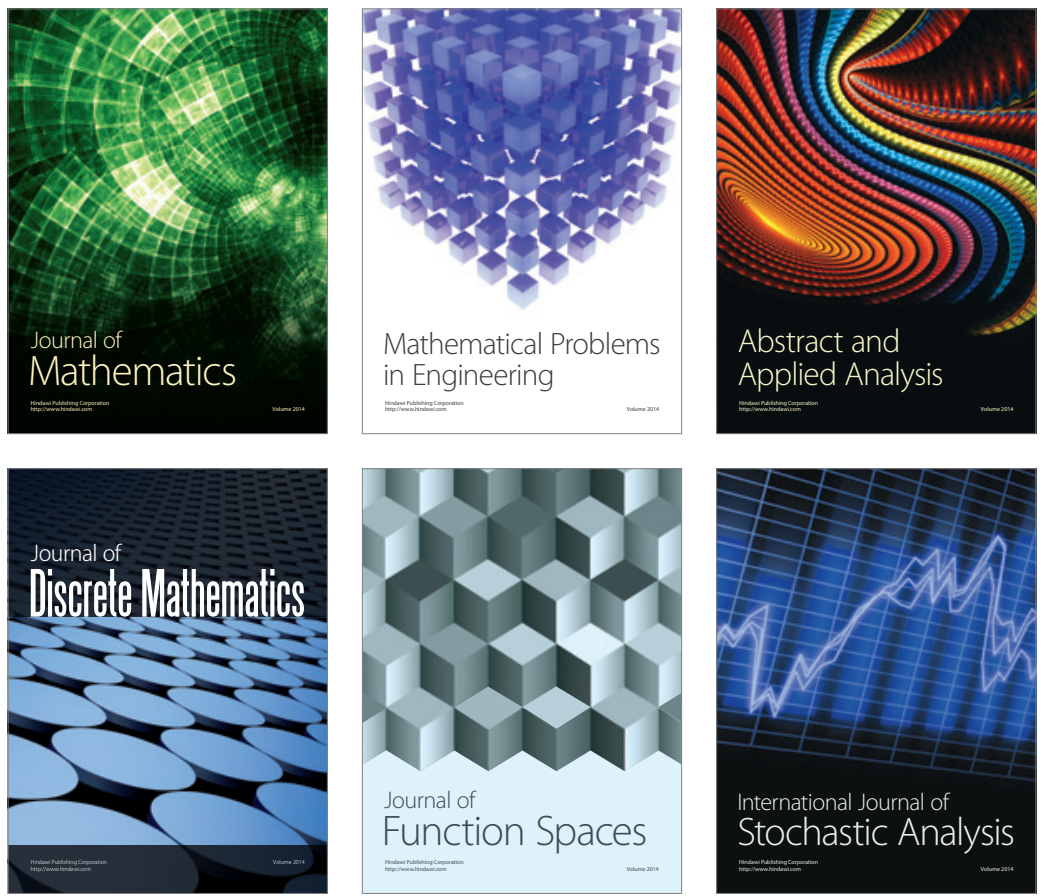

Journal of

Function Spaces

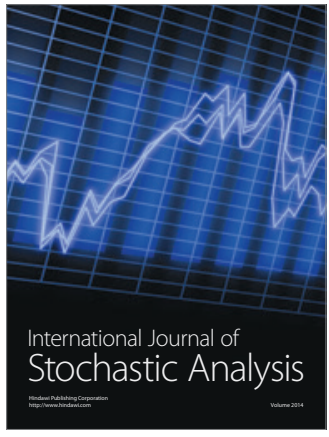

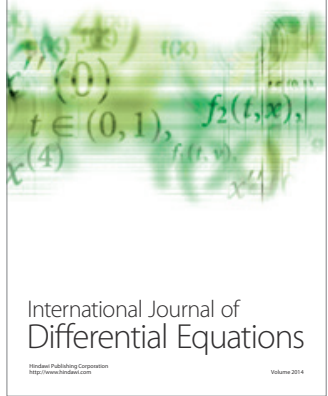
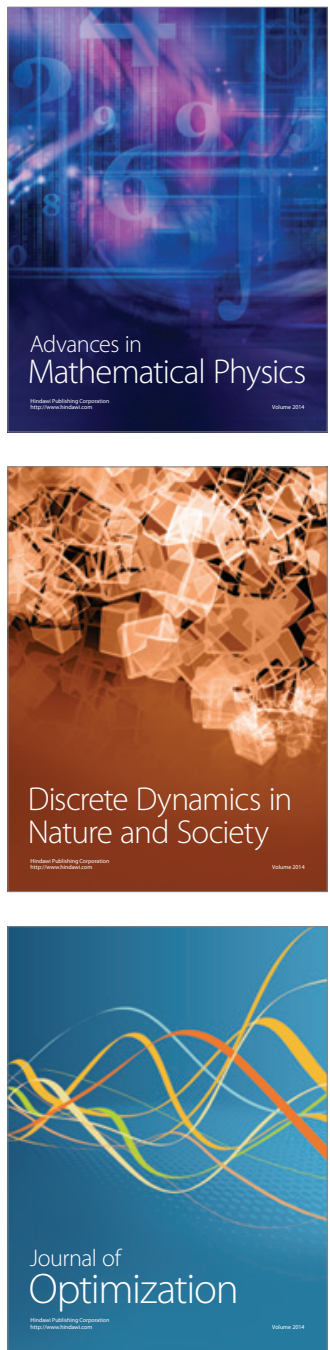